\title{
Histerectomia Pós-Parto de emergência em maternidade pública de cuidados de alto risco no estado do Amazonas
}

\author{
Postpartum Emergency hysterectomy in high-risk public maternity care in the state of \\ Amazonas
}

Histerectomía de emergencia posparto en atención pública de maternidad de alto riesgo en el estado de Amazonas

Lucas Simas de Souza $^{1 *}$, Andreia Ferreira de Souza1.

\begin{abstract}
RESUMO
Objetivo: Determinar a incidência, indicações, fatores de risco e complicações associadas com histerectomia pós-parto de emergência (HPPE)em maternidade pública de cuidados de alto risco no Estado do Amazonas. Métodos: $O$ estudo foi do tipo descritivo, observacional, corte transversal, por meio de prontuários médicos e de livros de registros de cirurgias foram identificados os casos de histerectomias pós-parto de emergência realizadas no período de janeiro de 2016 a dezembro de 2017. Os dados foram coletados no Hospital da cidade de Manaus-Amazonas. Resultados: No período de 2016 a 2017, houve 16.978 partos no da cidade de Manaus-Amazonas, 6.873 (40,5\%) corresponderam a cesarianas e 10.105 (59,5\%) a partos vaginais. Ocorreram 14 casos de histerectomia pós-parto de emergência, representando uma incidência de 0,82\1.000 partos; a indicação mais comum foi atonia uterina $50 \%$, seguida de acretismo placentário $29 \%$ e placenta prévia $21 \%$. Conclusão: A atonia uterina foi a indicação mais comum de HPPE em nossa Maternidade e nossas taxas de histerectomia após partos cesarianos mostraram-se nove vezes maiores às que se seguiram após um parto vaginal, logo, vemos a importância da adoção de práticas obstétricas de incentivo ao parto vaginal, especialmente após o primeiro parto cesariano, o que poderia minimizar riscos para o parto atual e consequentemente diminuir nossas taxas de HPPE.
\end{abstract}

Palavras-Chave: Histerectomia, Hemorragia Pós-parto, Parto Normal, Cesárea, Mortalidade Materna.

\begin{abstract}
Objective: To determine the incidence, indications, risk factors, and complications associated with emergency postpartum hysterectomy (HPPE) in a public maternity hospital for high-risk care in the state of Amazonas. Methods: This was a descriptive, observational, cross-sectional study. , through medical records and surgery record books, cases of emergency postpartum hysterectomies performed from January 2016 to December 2017 were identified. Data were collected at the Manaus-Amazonas Hospital. Results: From 2016 to 2017, there were 16,978 deliveries in the city of Manaus-Amazonas, 6,873 (40.5\%) corresponded to cesarean sections and 10,105 (59.5\%) to vaginal deliveries. There were 14 cases of postpartum hysterectomy. of emergency, representing an incidence of $0.82 \backslash 1,000$ deliveries; the most common indication was $50 \%$ uterine atonia followed by $29 \%$ placental accretism and $21 \%$ placenta previa. Conclusion: Uterine atony was the most common indication for HPPE in our Maternity and our hysterectomy rates after cesarean deliveries were nine times higher. larger than those following a vaginal delivery, therefore, we see the importance of adopting obstetric practices to encourage vaginal delivery, especially after the first cesarean delivery, which could minimize risks to current delivery and consequently lower our HPPE rates
\end{abstract}

Keywords: Hysterectomy, Postpartum Hemorrhage, Normal Birth, Cesarean Section, Maternal Mortality.

1Universidade do Estado do Amazonas - Manaus -AM. *E-mail: cearapossivel@msn.com 


\section{RESUMEN}

Objetivo: Determinar la incidencia, indicaciones, factores de riesgo y complicaciones asociadas con la histerectomía posparto de emergencia (HPPE) en un hospital público de maternidad para atención de alto riesgo en el estado de Amazonas. Métodos: Estudio descriptivo, observacional y transversalm através de registros médicos y libros de cirugía, se identificaron casos de histerectomías posparto de emergencia realizadas entre enero de 2016 y diciembre de 2017. Los datos se recopilaron en el Hospital ManausAmazonas. Resultados: De 2016 a 2017, hubo 16.978 partos en la ciudad de Manaus-Amazonas, 6.873 (40,5\%) correspondieron a cesáreas y 10.105 (59,5\%) a partos vaginales. Hubo 14 casos de histerectomía posparto. de emergencia, que representa una incidencia de 0,82 \1,000 entregas; la indicación más común fue $50 \%$ de atonía uterina, seguida de $29 \%$ de acretismo placentario y $21 \%$ de placenta previa. Conclusión: la atonía uterina fue la indicación más común de HPPE en nuestra sala de maternidad y nuestras tasas de histerectomía después de un parto por cesárea fueron nueve veces más altas. por lo tanto, vemos la importancia de adoptar prácticas obstétricas para fomentar el parto vaginal, especialmente después del primer parto por cesárea, lo que podría minimizar los riesgos para el parto actual y, en consecuencia, reducir nuestras tasas de HPPE.

Palabras-clave: histerectomía, hemorragia posparto, rutas del parto, parto vaginal y cesárea, mortalidad materna.

\section{INTRODUÇÃO}

A histerectomia pós-parto de emergência (HPPE) é um procedimento operatório que consiste na retirada do útero por via abdominal, realizada após um parto cesariano ou vaginal. É considerada uma cirurgia de grande porte, reservada a situações de ineficácia dos métodos conservadores para controle de hemorragia uterina (CHRISTOPOULOS P et al., 2011; IHAB AS et al., 2014).

Carroli $\mathrm{G}$ et al, (2008) apontaram que $2 \%$ a $11 \%$ dos nascimentos cursam com hemorragias pós-parto, e que existe uma grande variação entre os países, por diferentes cenários de cuidados obstétricos, e que somente nos Estados Unidos (EUA) representam $11 \%$ a $13 \%$ das mortes maternas. A hemorragia uterina segundo Haeri S e Dildy GA (2012), constitui uma das principais causas de mortalidade materna, a par da pré-eclâmpsia e infecção, responsável por 150.000 óbitos por ano em todo o mundo, segundo a Organização Mundial da Saúde (OMS), onde a metade dessas mortes ocorrem no período pós-parto, sendo a maioria nos países em desenvolvimento (MOUSA HA, WALKINSHAW S., 2001; KHAN KS et al., 2006).

Historicamente, foi em 1876, na cidade italiana de Paiva, que Eduardo Porro realizou a primeira histerectomia obstétrica bem-sucedida, em que tanto a mãe quanto a criança sobreviveram, e desde então se tornou um importante procedimento de emergência para controle da hemorragia (TODMAN DEP, 2006).

Mundialmente sua incidência é muito variável, alcança 0,2 casos por 1.000 partos em países desenvolvidos como a Noruega e 6,2 casos por 1.000 partos em países em desenvolvimento como a Nigéria, os Estados Unidos relatam incidência de 0,77 a 2,7 histerectomias por 1.000 partos (OMOLE- OHONSI A; OLAYINKA HT, 2012; OBIECHINA NJ et al., 2012).

Imudia AN et al, (2009), Rossi AC et al, 2010, Cromi A et al, (2016) e Campbell OM et al, (2016)apontaram fatores de risco para histerectomia pós-parto, dentre eles a multiparidade, partos cesáreos repetidos, gestação múltipla, placenta prévia, acretismo placentário, pré-eclâmpsia, idade materna avançada, sangramento anteparto, hemorragia pós-parto, distúrbios hemorrágicos e o uso de tecnologias de reprodução assistida.

Glaze S et al, (2008), observaram que, duas décadas atrás, as indicações mais comuns de HPPE consistiam em atonia uterina e rotura uterina. Recentemente, estudos em Portugal, Estados Unidos, Índia, Turquia, Dubai têm relatado um incremento no número de cesarianas e aumento na taxa de placentação anômala, placenta acreta e placenta prévia, consideradas as causas mais frequentes para o procedimento 
atualmente, principalmente em países desenvolvidos (ANSHUJA S et al, 2017; ABDULRAZZAQ MT et al, 2018).

Os estudos de Kastner ES et al, (2002); Francois K et al, (2005), Kazi S, (2018) mostraram uma estreita associação de HPPE e significativa morbimortalidade pós-operatória, perdas severas de sangue, risco de terapia transfusional e complicações operatórias.

Yucel O et al, (2005); Selo-Ojeme DO et al, (2005) reportaram que as complicações mais frequentes após HPPE são a necessidade de grandes transfusões, cuidados em Unidade de Terapia Intensiva (UTI), as coagulopatias (CIVD), lesões de trato urinário, necessidade de reintervenção cirúrgica, hemorragia persistente e morbidade febril.

Ramilo L et al, (2015); Friedman AM et al, (2016) relataram que no ciclo gestacional a histerectomia deve ser encarada como um procedimento de exceção, mas que não deve ser protelado na vigência de quadros hemorrágicos graves e refratários a condutas conservadoras. Consideram a HPPE um procedimento "salvavidas" importante no controle da hemorragia pós-parto e fundamental para se evitar a morte materna.

Assim a HPPE é um procedimento utilizado para administrar hemorragias obstétricas com risco de vida elou sepse uterina. Em contraste, o aumento global das taxas de cesarianas pode levar a uma maior incidência de patologias placentárias e, portanto, incremento na incidência de histerectomia, e sendo assim, o objetivo foi discorrer e abordar sobre histerectomia pós-parto de emergência em maternidade pública de cuidados de alto risco no Estado do Amazonas.

\section{MÉTODOS}

O estudo foi do tipo descritivo, observacional, corte transversal por meio de prontuários médicos e de livros de registros de cirurgias foram identificados os casos de histerectomias pós-parto de emergência realizadas no período de janeiro de 2016 a dezembro de 2017.

Os dados foram coletados em um hospital da cidade de Manaus-Amazonas. A unidade possui 143 leitos de Alojamento Conjunto (ALCON); 15 leitos de pré-parto; 6 leitos de parto normal; 5 leitos de UTI materna; 25 leitos de UTI neonatal. O projeto foi submetido ao Comitê de Ética e Pesquisa da Universidade do Estado do Amazonas (UEA) e atendeu às normas da Resolução 466/2012 do Conselho, sob o número de autorização CAAl: 06519619.80000 .5016$.

Foi solicitado ao comitê a dispensa do Termo de Consentimento Livre e Esclarecido por se tratarem de dados secundários. A amostra foi composta por 110 prontuários de mulheres que foram submetidas a laparotomia de emergência coletados em um hospital da cidade de Manaus-Amazonas. no período de janeiro de 2016 a dezembro de 2017.

Os Critérios de Inclusão foram: Pacientes submetidas a histerectomias pós-partos de emergência ocorridas na Maternidade, no período de 1 de janeiro de 2016 a 31 de dezembro de 2017; Pacientes com via de parto vaginal ou cesariana.

E para os critérios de exclusão: Casos em que o preenchimento dos prontuários estava inadequado e /ou danificado pelas condições ambientais e de armazenamento (ilegíveis, ausência de dados ou dados incompletos), além disso as que pacientes que foram submetidas à HPPE em outra instituição e encaminhada à Maternidade apenas para internação na Unidade de Terapia Intensiva (UTI).Paciente que foi submetida à HPPE com idade gestacional menor que 22 semanas ou peso do feto menor que $500 \mathrm{~g}$.

A incidência de HPPE ocorridas em um hospital da cidade de Manaus-Amazonas, no período de janeiro de 2016 a dezembro de 2017, foi determinada através do número de histerectomias realizadas no período de estudo e o total de partos vaginais e cesarianos ocorridos no mesmo período.

Quanto ao perfil sociodemográfico as variáveis analisadas foram: profissão; escolaridade; estado civil que definimos como solteira (sem companheiro/a) e casada (presença de companheiro/a); idade com corte de 25 anos; corlraça que definimos como branca, parda e negra e a naturalidade. 
Em relação ao perfil clínico-epidemiológico foram analisadas o número de consultas pré-natais; idade gestacional que iniciou o pré-natal; paridade; antecedentes obstétricos; comorbidades associadas; idade gestacional, exame físico e obstétrico no dia de admissão em um hospital da cidade de Manaus-Amazonas. Quanto a identificação dos fatores de risco para HPPE das pacientes que realizaram o procedimento, as variáveis analisadas foram: placenta prévia; acretismo placentário; distúrbios de coagulação; gravidez gemelar; cesariana atual elou anterior; hemorragia pós-parto; grande multípara; iteratividade (3 cesáreas ou mais); distúrbios hipertensivos na gestação (pré-eclâmpsia, hipertensão super ajuntada); idade materna avançada; uso de anticoagulante; descolamento prematuro de placenta; trabalho de parto prolongado e corioamnionite.

Para identificação das causas das hemorragias pós-parto em mulheres que realizaram histerectomia foram analisadas as variáveis: tônus uterino, trauma do canal de parto elou útero, tecido placentário e suas características de implantaçãolretenção, distúrbios de coagulação, anemia na gestação, parto por fórceps, parto induzido, parada de progressão, uso de episiotomia, lacerações de terceiro e quarto graus; para estimar o tempo desprendido para a realização da histerectomia foram utilizados os boletins cirúrgicos e anestésicos e determinação do tempo foi dada em horas e minutos.

Em relação as identificações das complicações pós-operatórias relacionadas a histerectomia de emergência as variáveis analisadas foram: lesão de bexiga, lesão de alça intestinal, lesão de grandes vasos, lesão ureteral, transfusão sanguínea, internação em UTI, necessidade de nova abordagem cirúrgica, sepse, coagulação intravascular disseminada (CIVD), choque hipovolêmico, óbito e outras. Na avaliação do tempo de hospitalização das pacientes histerectomizadas foi feita comparando o tipo de histerectomia realizada se total ou subtotal e o tempo de hospitalização em dias. Para composição de todas as variáveis utilizadas no estudo foi elaborada uma ficha de coleta de dados para registros das informações existentes nos prontuários físicos elou eletrônicos, livros de cirurgias referentes ao período de tempo que compreendeu o referido.

Os dados foram apresentados por meio de gráficos e tabelas através do cálculo das frequências absolutas simples e relativas para os dados categóricos. $\mathrm{Na}$ análise dos dados quantitativos, quando se aceitou a hipótese de normalidade por meio do teste de Shapiro-Wilk, foi calculada a média e o desvio-padrão (Dp), no entanto, quando rejeitada a hipótese de normalidade, foi calculada a mediana e os quartis Q1(25\%) e Q3(75\%). Na comparação das médias foi aplicado o teste $t$-student e o software utilizado na análise dos dados foi o programa Epi Info versão 7.2 para windows que foi desenvolvido e distribuído gratuitamente pelo Centro de Prevenção e Controle de Doenças Norte-Americano - CDC (www.cdc.gov/epiinfo) e o nível de significância fixado nos testes estatísticos foi de $5 \%$.

\section{RESULTADOS E DISCUSSÃO}

No período de 2016 a 2017, houve 16.978 partos na Maternidade em um hospital da cidade de ManausAmazonas, $6.873(40,5 \%)$ corresponderam a cesarianas e $10.105(59,5 \%)$ a partos vaginais. Ocorreram 14 casos de histerectomia pós-parto de emergência, 12 pós-cesarianas e 2 pós-partos vaginais, representando uma incidência de0, 8211.000partos (Tabela 1). Quando analisados os partos cesarianos (PC) a incidência foi de 1,74\1.000 nascimentos e para partos vaginais (PV) a incidência foi de 0,19\1.000. A incidência de HPPE foi 9,1 vezes maior após o parto cesariano destacados na Tabela 1.

Tabela 1 - Taxa de partos e histerectomia pós-parto (HPPE) em gestantes submetidas aos procedimentos em uma maternidade de referência no período de 2016 a 2017, Manaus - AM.

\begin{tabular}{lrcccc}
\hline TIPO DE PARTO & № de partos & $\begin{array}{c}\text { Taxa de partos } \\
(\%)\end{array}$ & № de HPPE & $\begin{array}{c}\text { Taxa de HPPE } \backslash \\
(\mathbf{1 0 0 0} \text { partos) }\end{array}$ \\
\hline CESÁREA & 6.873 & 40,5 & 12 & 1,74 \\
VAGINAL & 10.105 & 59,5 & 2 & 0,19 \\
TOTAL & 16.978 & 100,0 & 14 & 0,82 \\
\hline
\end{tabular}

Fonte: Souza LS, Souza AF, 2016 e 2017. 
A idade média das pacientes histerectomizadas foi 27,5 \pm 6,3 anos (amplitude 17-35), sendo 92,9\% das mulheres donas de casa e $7,1 \%$ estudantes, quanto a escolaridade $64,3 \%$ estudou somente o ensino fundamental e $35,7 \%$ chegou ao ensino médio. A procedência das gestantes $42,9 \%$ eram da zona rural e $57,1 \%$ procedentes de Manaus, o status civil de casada configurou em $57 \%$ das grávidas e $42,9 \%$ eram solteiras, conforme tabela 2.

Tabela 2 - Distribuição segundo os dados sociodemográficos das gestantes submetidas a histerectomia em uma maternidade de referência no período de 2016 a 2017, Manaus - AM.

\begin{tabular}{|c|c|c|}
\hline Variáveis $(n=14)$ & $f_{i}$ & $\%$ \\
\hline \multicolumn{3}{|l|}{ Idade (anos) } \\
\hline$<25$ & 6 & 42,9 \\
\hline$\geq 25$ & 8 & 57,1 \\
\hline Média $\pm \mathrm{Dp}$ & $27,5 \pm 6,3$ & \\
\hline Amplitude & $17-35$ & \\
\hline \multicolumn{3}{|l|}{ Profissão } \\
\hline Do lar & 13 & 92,9 \\
\hline Estudante & 1 & 7,1 \\
\hline \multicolumn{3}{|l|}{ Escolaridade } \\
\hline Ensino fundamental & 9 & 64,3 \\
\hline Ensino médio & 5 & 35,7 \\
\hline \multicolumn{3}{|l|}{ Estado civil } \\
\hline Casada & 8 & 57,1 \\
\hline Solteira & 6 & 42,9 \\
\hline \multicolumn{3}{|l|}{ Procedência } \\
\hline Manaus & 8 & 57,1 \\
\hline Zona rural & 6 & 42,9 \\
\hline
\end{tabular}

$\mathrm{f}_{\mathrm{i}}=$ frequência absoluta simples; $\mathrm{Dp}=$ desvio-padrão.

Fonte: Souza LS, Souza AF, 2016 e 2017.

A cesariana foi a indicação de via de parto em $85,7 \%$ das mulheres histerectomizadas. As indicações foram: 2 por descolamento prematuro de placenta com evolução para óbito fetal em um dos casos; 3 indicações por iteratividade em que 2 mulheres estavam na quarta cesárea e 1 realizava o procedimento pela sexta vez consecutiva; 2 indicações por apresentação pélvica; 1 por cesariana há menos de dois anos;1 por pré-eclâmpsia grave em idade gestacional de 33 semanas com placenta prévia total; 1 por falha de indução de trabalho de parto em feto morto de 34 semanas; 1 por macrossomia fetal de $4.490 \mathrm{~g}$ com diabetes gestacional. As características clínico-epidemiológicas estão demonstradas na Tabela 3. 
Tabela 3 - Distribuição segundo os dados das gestações, partos e abortos das gestantes submetidas a histerectomia em uma maternidade de referência no período de 2016 a 2017, Manaus - AM.

\begin{tabular}{|c|c|c|}
\hline Variáveis $(n=14)$ & $\mathbf{f}_{\mathrm{i}}$ & $\%$ \\
\hline \multicolumn{3}{|l|}{ Número de gestações } \\
\hline 1 & 1 & 7,1 \\
\hline 2 & 2 & 14.3 \\
\hline 3 & 4 & 28,6 \\
\hline$>3$ & 7 & 50,0 \\
\hline Média \pm Dp & $4,2 \pm 2,2$ & \\
\hline Amplitude & $1-8$ & \\
\hline \multicolumn{3}{|l|}{ Partos normais } \\
\hline 0 & 6 & 42,9 \\
\hline 1 & 1 & 7,1 \\
\hline 2 & 3 & 21,4 \\
\hline$>2$ & 4 & 28,6 \\
\hline Média \pm Dp & $1,9 \pm 2,4$ & \\
\hline Amplitude & $0-8$ & \\
\hline \multicolumn{3}{|l|}{ Partos cesáreos } \\
\hline 0 & 2 & 14,3 \\
\hline 1 & 4 & 28,6 \\
\hline 2 & 4 & 28,6 \\
\hline$>2$ & 4 & 28,6 \\
\hline Média \pm Dp & $2,0 \pm 1,8$ & \\
\hline Amplitude & $0-6$ & \\
\hline \multicolumn{3}{|l|}{ Abortos } \\
\hline 0 & 11 & 78,6 \\
\hline 1 & 2 & 14,3 \\
\hline 2 & 1 & 7,1 \\
\hline Média \pm Dp & $0,3 \pm 0,6$ & \\
\hline \multicolumn{3}{|l|}{ Indicação cesárea } \\
\hline Iteratividade & 3 & 21,4 \\
\hline Descolamento Prematuro de Placenta & 2 & 14,3 \\
\hline Apresentação pélvica & 2 & 14,3 \\
\hline Falha de indução & 2 & 14,3 \\
\hline Pré-eclâmpsia & 1 & 7,1 \\
\hline Cesárea a menos de 2 anos & 1 & 7,1 \\
\hline Macrossomia fetal & 1 & 7.1 \\
\hline Nenhuma & 2 & 14,3 \\
\hline
\end{tabular}

Fonte: Souza LS, Souza AF, 2016 e 2017. 
Foi observado que $78,6 \%$ das mulheres apresentavam pelo menos dois fatores de risco para HPPE. A cicatriz uterina por cesariana anterior presente em $57 \%$ dos casos, seguida de multiparidade em $50 \%$, além de acretismo placentário $28,6 \%$ e placenta prévia $21,4 \%$ descritos na tabela 4.

Tabela 4 - Distribuição segundo os fatores de risco para HPP, etiologia e medidas de controle hemorrágico nas gestantes submetidas a histerectomia em uma maternidade de referência no período de 2016 a 2017 , Manaus - AM.

\begin{tabular}{|c|c|c|}
\hline Variáveis $(n=14)$ & $f_{i}$ & $\%$ \\
\hline \multicolumn{3}{|l|}{ Fatores de risco para HPP } \\
\hline Grande multípara & 7 & 50,0 \\
\hline Trabalho de parto prolongado & 4 & 28,6 \\
\hline Placenta acreta & 4 & 28,6 \\
\hline Placenta previa & 3 & 21,4 \\
\hline Descolamento prematuro de placenta & 3 & 21,4 \\
\hline Iteratividade & 3 & 21,4 \\
\hline Pré-eclâmpsia & 3 & 21,4 \\
\hline Parada de progressão & 2 & 14,3 \\
\hline Anemia gestacional & 2 & 14,3 \\
\hline Retenção placentária & 1 & 7,1 \\
\hline \multicolumn{3}{|l|}{ Status do risco para HPP } \\
\hline Alto risco & 9 & 64,3 \\
\hline Médio risco & 2 & 14,3 \\
\hline Baixo risco & 3 & 21,4 \\
\hline \multicolumn{3}{|l|}{ Etiologia da HPP } \\
\hline Tecido & 6 & 42,8 \\
\hline Tônus & 8 & 57,2 \\
\hline Medidas de controle hemorrágico & 9 & 64,3 \\
\hline HPPE imediata & 5 & 35,7 \\
\hline
\end{tabular}

Fonte: Souza LS, Souza AF, 2016 e 2017. 
A indicação mais comum de HPPE foi atonia uterina em 7 casos $(50,0 \%)$, seguida de acretismo placentário 4 casos $(28,6 \%)$ e placenta prévia 03 casos $(21,4 \%)$ (Gráfico 5 ).

Gráfico 5. Indicações de histerectomias pós-parto de emergênciamaternidade de referência no período de 2016 a 2017, Manaus - AM.

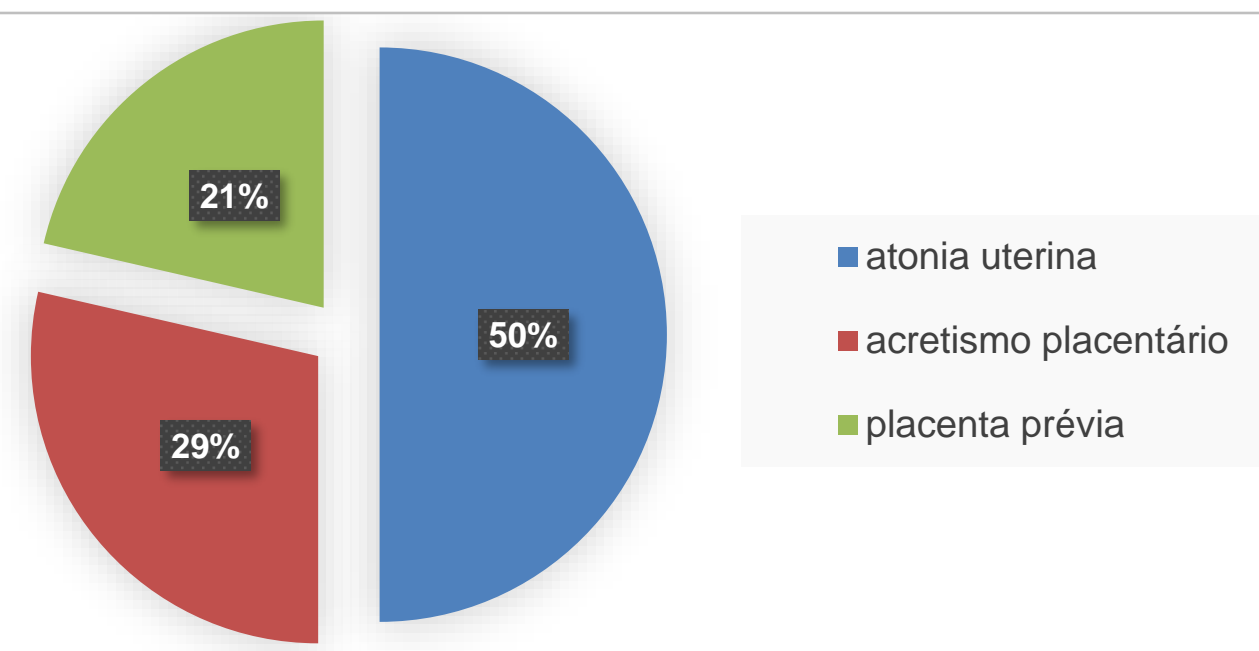

Fonte: Souza LS, Souza AF, 2016 e 2017.

A histerectomia total foi realizada como técnica cirúrgica em 4 mulheres $(28,6 \%)$ e a subtotal em 10 delas (71,4\%). O tempo médio por cirurgia foi de 129,3 \pm 37,3 minutos, amplitude de 60-180 min; (Gráfico 6).

Gráfico 6 - Média de histerectomia realizada em maternidade de referência no período de 2016 a 2017, Manaus - AM.

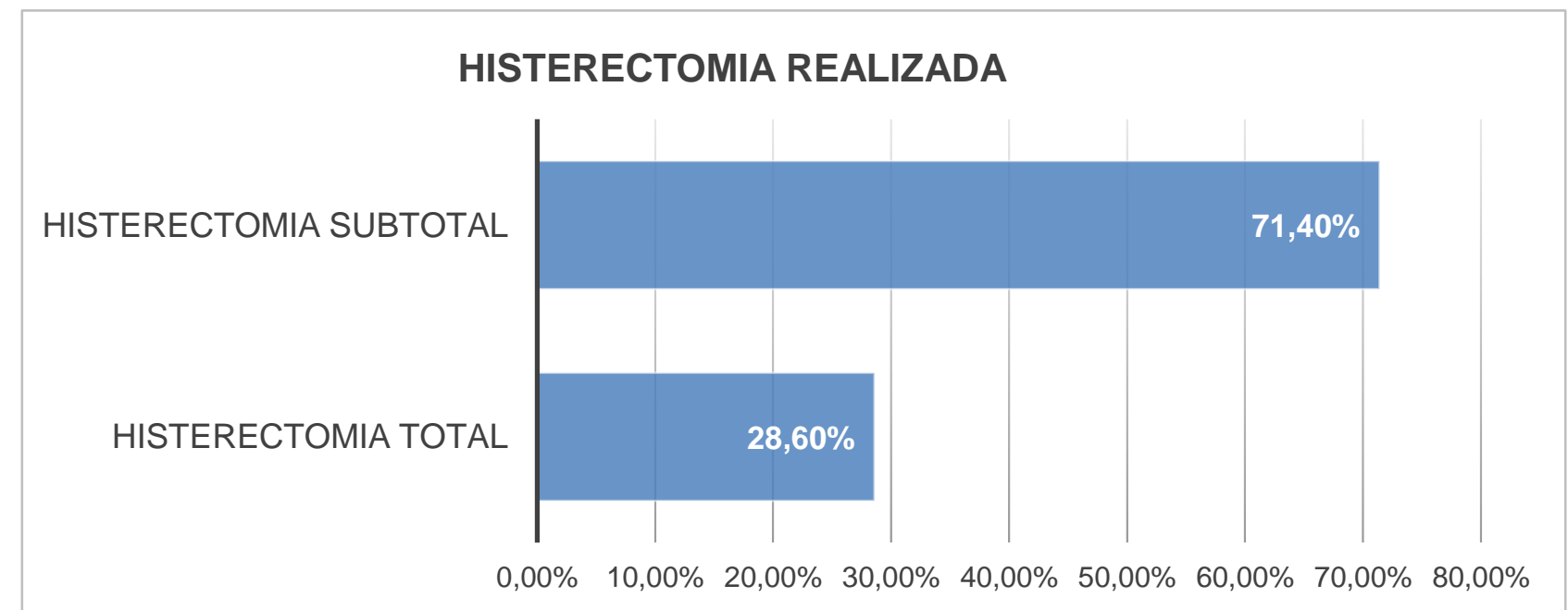

Fonte: Souza LS, Souza AF, 2016 e 2017.

O estudo demonstrou que as pacientes evoluíram com mais de uma complicação pós-operatória, como necessidade de UTI presente em 100\% dos casos; onde 12 mulheres (85,7\%) precisaram de terapia transfusional e 7 mulheres (50\%) evoluíram com choque hipovolêmico. Outras complicações observadas foram anexectomia (28\%) 4 casos; 3 mulheres $(21,4 \%)$ precisaram de nova abordagem cirúrgica; 2 mulheres $(14,3 \%)$ apresentaram sepse; $2(14,3 \%)$ hematoma subaponeurótico e 1 caso de óbito materno $(7,1 \%)$ após choque hipovolêmico, descritos na tabela 7. 
Tabela 7 - Distribuição segundo as complicações no pós-operatório, concentrado de hemácias $(\mathrm{CH})$, plasma fresco congelado (PFC) e plaquetas (PLT) nas gestantes submetidas a histerectomia em uma maternidade de referência no período de 2016 a 2017, Manaus - AM.

\begin{tabular}{lrr}
\hline Variáveis $(\mathbf{n}=\mathbf{1 4})$ & $f_{i}$ & $\%$ \\
\hline Complicações no pós-operatório & 14 & 100,0 \\
UTI & 12 & 85,7 \\
Hemotransfusão & 7 & 50,0 \\
Choque hipovolêmico & 4 & 28,6 \\
Anexectomia & 3 & 21,4 \\
Relaparotomia & 2 & 14,3 \\
Sepse & 2 & 14,3 \\
Lesão vesical & 2 & 14,3 \\
Hematoma sub aponeurótico & 2 & 7,1 \\
Óbito & 1 &
\end{tabular}

$\mathrm{CH}$

Média $\pm \mathrm{Dp}$

$4,8 \pm 2,7$

Amplitude

$0-10$

PFC

$Q_{1}$ - Mediana - $Q_{3}$

$0,0-0,0-2,0$

Amplitude

$0-4$

PLT

Q1 - Mediana - $Q_{3}$

$0,0-0,0-2,0$

Amplitude

$0-4$

$\overline{f_{i}}=$ frequência absoluta simples; $D p=$ desvio-padrão. Concentrado de hemácias $(\mathrm{CH})$, plasma fresco congelado (PFC) e plaquetas (PLT).

Fonte: Souza LS, Souza AF, 2016 e 2017.

O tempo médio de internação hospitalar (DIH) foi de $8,9 \pm 4,1$ dias (mínimo de 1 e máximo de 16 dias). Avaliamos o tempo de internação em relação ao tipo de histerectomia(total e subtotal) chegando a uma média de 9,7 $\pm 3,9$ para histerctomia subtotal e de 7,0 $\pm 4,2$ para a histerectomia total com $\mathrm{p}^{*}$ de 0,279 para as duas variáveis, já quanto ao tipo de parto (cesariano e vaginal) observou-se que para o tipo de parto cesárea a média foi de $9,4 \pm 4,2$ e para o parto vaginal $6,0 \pm 1,4$ com $\mathrm{p}^{*} 0,289$ para ambos os partos, descritos na tabela 8. 
Tabela 8 - Comparação dia de internação hospitalar (DIH) em relação ao tipo de histerectomia em gestantes submetidas ao procediemento em uma maternidade de referência no período de 2016 a 2017, Manaus - AM.

\begin{tabular}{lrrc}
\hline Variáveis & Média & DP & $\mathrm{p}^{*}$ \\
\hline Histerectomia & & & 0,279 \\
Subtotal & 9,7 & 3,9 & \\
Total & 7,0 & 4,2 & \\
\hline D $=$ desvio-padrão: * Teste t-student & & &
\end{tabular}

$\mathrm{DP}=$ desvio-padrão; * Teste $t$-student.

Fonte: Souza LS, Souza AF, 2016 e 2017.

A média de partos na Maternidade está em torno de 8.500 por ano. Durante os dois anos do estudo ocorreram 14 histerectomias pós-parto de emergência (HPPE), período em que houve 16.978 partos, correspondendo a uma incidência de 0,82 por 1.000 nascimentos, o que é similar a estudos reportados em Portugal (0,6\1.000) Ramilo L et al, (2015), Estados Unidos (0,77\1.000).

A idade materna variou de 17 a 35 anos, com média de idade de 27,5 anos, idade gestacional média de 36,8 semanas e a média de gestações de 4 filhos. Nenhuma paciente estava inserida no mercado de trabalho, $92 \%$ delas estudaram apenas ensino fundamental, a metade era procedente da zona rural, o que sugere uma população de baixo nível socioeconômico. A meta-análise de Akker TV, et al. (2016) que incluiu 129 estudos sobre HPPE pelo mundo mostrou uma maior prevalência de histerectomia obstétrica de emergência em populações de baixa renda de países em desenvolvimento. No seu estudo a idade média das pacientes (26,2 anos) a idade gestacional média (37 semanas) e o percentual de multíparas foi semelhante ao que o estudo encontrou.

Um importante estudo de Rossi AC et al., (2010) de 24 artigos, que incluíram 981 casos de HPPE, mostraram que as mulheres com maior fator de risco de histerectomia são as multíparas, as que tiveram cesariana em uma gravidez anterior ou atual e as que apresentaram placenta anômala, (acreta elou prévia). $O$ estudo encontrou fatores de riscos semelhantes. Das 14 mulheres histerectomizadas 12 foram após parto cesariano (78,4\%),8 tinham cicatrizes de cesariana anterior (57\%), 7 mulheres eram multíparas (50\%), e 7 gestantes apresentavam placenta anômala (50\%).

À medida que o número de cesarianas aumenta, também aumentam as cicatrizes uterinas, expondo assim a grávida a chance maior de ruptura do útero, acretismo placentário, placenta previa e incidência de HPPE, e isso vem mudando as indicações de histerectomias nas últimas duas décadas, foi o que mostrou um estudo de Flood KM et al, (2009) em três hospitais obstétricos de Dublin, onde ocorreram 358 HPPE e a indicação por acretismo placentário aumentou de $5,4 \%$ passando para $46,5 \%$.

O acretismo placentário foi responsável por $28,6 \%$ das indicações (4) casos de HPPE, a atonia uterina foi a etiologia encontrada em50\% (7) casos, e placenta prévia em 21,4\% (3) casos. Nossas taxas de antecedentes de cesarianas em mulheres submetidas a HPPE são consideradas altas $57 \%$, semelhantes as demonstradas por Ibrahim M et al, (2014) e Sahin SL et al, (2014) em seus estudos, onde ultrapassavam $50 \%$ e 65\% respectivamente, porém a nossa maior indicação de HPPE foi por atonia uterina(50\%).

Quanto a complicações pós-operatórias a literatura reporta comumente uma maior necessidade de transfusões maciças por perdas hemáticas, necessidade de internação em unidades de terapia intensiva, lesões do trato urinário, coagulação intravascular disseminada CIVD, bem como a necessidade de reabordagem cirúrgica e casos de óbito materno, Selo-Ojeme DO et al, (2005); Yucel O et al, (2006); Rossi AC et al. (2010); Kazi S,(2018).

O estudo identificou em $100 \%$ das HPPE a necessidade de internação em UTI como a complicação mais comum, seguida de necessidade de terapia transfusional (85\%) e choque hipovolêmico (50\%), 3 casos de reabordagem cirúrgica $(21,4 \%)$, lesão vesical ocorreu em 2 casos (14\%) e tivemos 1 óbito materno (7\%) por choque hipovolêmico, o que é muito similar aos estudos supracitados. Estudos demonstraram taxas de 
mortalidade que variaram de $1 \%$ a $6 \%$ em todo o mundo e que a mortalidade materna após histerectomia foi alta em populações de baixa renda Rossi AC et al, (2010). No estudo de Obiechina NJ et al, (2018) na Nigéria a taxa de mortalidade pós HPPE encontrada foi de 31\%. O estudo de Kazi S (2018), no Paquistão encontrou taxa de mortalidade de 9,3\%, na Índia essa taxa foi 7,2\% como demostrou Anshuja S et al, 2017. Diante disso foi possível verificar os diferentes níveis de cuidados obstétricos entre os países, ainda que as intercorrências sejam muitas das vezes semelhantes, ainda há significativa diferença em seus percentuais e incidência.

\section{CONCLUSÃO}

Podemos enfatizar com este estudo a importância de se evitar partos cesáreos desnecessários, especialmente em primíparas, uma vez que nossas taxas de histerectomia após partos cesarianos mostraram-se nove vezes maiores às que se seguiram após um parto vaginal. Igualmente importante é a adoção de práticas obstétricas de incentivo ao parto vaginal, especialmente após o primeiro parto cesariano, o que poderia minimizar riscos para o parto atual, uma vez que partos cesarianos repetidos com exposição do útero a inúmeras cicatrizes podem elevar o risco de placentação anômala e conseqüentemente aumento dos riscos de se evoluir para HPPE.

\section{REFERÊNCIAS}

1. ABDULRAZZAQ MT, et al. Emergency peripartum hysterectomy in the Dubai health system: A fifteen-year experience. Turk J Obstet Gynecol.2018; 15:1-7.

2. AKER TV de et al. Prevalence, indications, risk indicators, and outcomes of emergency peripartum hysterectomy word wide. Obstet Gynecol, Dez Vol 6, 1281, 2016.

3. ANSHUJA S, et al. Emergency Peripartum Hysterectomy: Indications and Outcome in a Tertiary Care Setting. Journal of Clinical and Diagnostic Research. 2017;Mar, Vol-11(3): QC01-QC03.

4. CAMPBELL OM, et al Lancet Maternal Survival Series Steering Group. Strategies for reducing maternal mortality: getting on with what Works. Lancet. 2006:368 (9543):1284-99.

5. CARROLI G, et al. Epidemiology of postpartum haemorrhage: a systematic review. Best Pract Res Clin Obstet Gynaecol 2008;22(6):999-1012.

6. CHRISTOPOULOS $P$, et al. Obstetric hysterectomy: A review of cases over 16 years. Journal of Obstetrics and Gynaecology, February; 2011; 31(2): 139-141.

7. CROMI A, et al. Risk of peripartum hysterectomy in births after assisted reproductive technology. Fertil Steril. E pub Jun ; 2016;106(3):623.

8. FLOOD KM, et al. Changing trends in peripartum hysterectomy over the last 4 decades. Am J Obstet Gynecol. Jun; 2009(6):632.e1-6.

9. FRANCOIS K. Is peripartum hysterectomy more common in multiple gestations. Obstet Gynecol. 2005; 105:1369-2.

10. FRIEDMAN AM, et al. Population-based risk for peripartum hysterectomy during low-and moderate-risk delivery hospitalizations. Am J Obstet Gynecol. 2016; 215(5):640.

11. GLAZE S, et al. Peripartum hysterectomy: 1999 to 2006. Obstet Gynecol 2008.

12. HAERI S, DILDY GA. Maternal mortality from hemorrhage. Semin Perinatol 2012;36(1):48-55.

13. IBRAHIM M. Incidence, indications, and predictors of adverse outcomes of postpartum hysterectomies: 20 - year experience in a tertiary care centre. Journal of obstetrics and gyneaecology Canada. Jan, Vol 36:14-20, 2014.

14. IHAB SA, et al. Incidence of emergency peripartum hysterectomy in Ain-shams University Maternity Hospital, Egypt: a retrospective study. Arch Gynecol Obstet. May 2014.

15. IMUDIA NA, et al. Incidence, trends, risk factors, indications for, and complications associated with cesarean hysterectomy: a 17-year experience from a single institution. Arch Gynecol Obstet. 2009; 280:619.

16. KASTNER ES, et al. Emergency peripartum hysterectomy: experience at a community teaching hospital. Obstet Gynecol 2002; 99:971-5.

17. KAZI S. Emergency peripartum hysterectomy: A great obstetric challenge. Pak J Med Sci. 2018;34(6):1567-1570.

18. KHAN KS, et al. WHO analysis of causes of maternal death: a systematic review. Lancet 2006;367 (9516):10661074.

19. MOUSA HA, WALKINSHAW S. Major postpartum haemorrhage. Curr Opin Obstet Gynecol 2001;13(6):595-603.

20. OBIECHINA NJ, et al. Emergency peripartum hysterectomy in Nnewi, Nigeria: a 10-years review. Niger J Clin Pract. 2012; 15(2):168-171. 
21. OMOLE-OHONSI A, OLAYINKA HT. Emergency peripartum hysterectomy in a developing country. J Obstet Gynaecol Can. 2012;34(10):954-60.

22. RAHMAN J, et al. Emergency obstetric hysterectomy in a university hospital: A 25-year review. J Obstet Gynaecol. 2008;28(1):69-72.

23. RAMILO L, et al. Histerectomia pós-parto: revisão de 15 anos. Acta Obstet Ginecol Port; 2015;9(1):16-22.

24. ROSSI AC, et al. Emergency postpartum hysterectomy for uncontrolled postpartum bleeding: a systematic review. Obstet Gynecol. 2010;115(3):637.

25. SAHIN SL et al. Emergency peripartum hysterectomy: our 12-years experience. Arch Gynecol Obstet 2014;289(5):953-958.

26. SELO-OJEME DO, et al. Emergency peripartum hysterectomy in a tertiary London hospital. Arch Gynecol Obstet 2005;271(2):154-159.

27. TODMAN DEP (1842 - 1902) and the Development of Caesarean Section: A Reappraisal. The Internet Journal of Gynecology and Obstetrics. Vol. 7 Number 2. 2006.

28. YUCEL O, et al. Emergency peripartum hysterectomy: a 9-year review. Arch Gynecol Obstet 2006;274(2):84-87. 